\title{
Analytical Wideband Model for Strip/Slit Gratings Loaded With Dielectric Slabs
}

\author{
Raúl Rodríguez-Berral, Carlos Molero, Francisco Medina, Fellow, IEEE, and Francisco Mesa, Senior Member, IEEE
}

\begin{abstract}
This paper presents a fully analytical model to determine the transmission and reflection properties of planar 1-D distributions of metal strips or slits made in thin metal screens. In contrast with other analytical or quasi-analytical approaches, the formulation incorporates the presence of dielectric slabs and is valid over a wide frequency band, from the long wavelength limit to the grating lobes operation. The model has been adapted to the case where two 1-D planar grids are stacked or a single grid is printed on a grounded substrate. In these cases, the model rigorously takes into account higher order mode interaction between the two stacked arrays of strips/slits or with the ground plane. Oblique incidence and both $\mathrm{TE}$ and $\mathrm{TM}$ polarizations have been considered. The analytical results show a good agreement with those computed by high-performance numerical methods, accounting for very fine details of extremely complicated transmission/reflection spectra. These results are of straightforward application to a variety of practical situations from microwaves to the terahertz regime. The present methodology can still be useful at higher frequencies provided that adequate models of the planar conductors are incorporated. In general, the model provides physical insight on the nature of the expected spectra and facilitates the design of devices based on planar metallic gratings.
\end{abstract}

Index Terms-Diffraction gratings, equivalent-circuit model, extraordinary and conventional transmission/reflection, impedance matching.

\section{INTRODUCTION}

$\mathbf{T}$ HE ANALYSIS of the electromagnetic response of periodic distributions of metallic planar scatterers (or planar apertures made in a metallic flat surface) has been a topic of interest for decades. This type of structure finds applications at very different frequency ranges, from the microwave and millimeter-wave bands (frequency-selective surfaces [1], polarizers [2], [3], artificial magnetic conductors [4], high-impedance surfaces [5], or partially reflective surfaces [6], just to mention a few examples) to the optical regime

Manuscript received July 10, 2012; revised September 24, 2012; accepted September 25, 2012. Date of publication November 21, 2012; date of current version December 13, 2012. This work was supported by the Spanish Ministerio de Ciencia e Innovación and European Union FEDER funds under Project TEC2010-16948 and Project Consolider CSD2008-00066 and by the Spanish Junta de Andalucía under Project P09-TIC-4595. This paper is an expanded paper from the IEEE MTT-S International Microwave Symposium, Montreal, QC, Canada, June 17-22, 2012.

R. Rodríguez-Berral, C. Molero, and F. Mesa are with the Microwaves Group, Department of Applied Physics 1, Escuela Técnica Superior de Ingeniería Informática, University of Seville, 41012-Seville, Spain (e-mail: rrberral@us.es; mesa@us.es; carmoljim@alumn.us.es).

F. Medina is with the Faculty of Physics, Department of Electronics and Electromagnetism, University of Seville, 41012-Seville, Spain (e-mail: medina@us. es).

Color versions of one or more of the figures in this paper are available online at http://ieeexplore.ieee.org.

Digital Object Identifier 10.1109/TMTT.2012.2224668

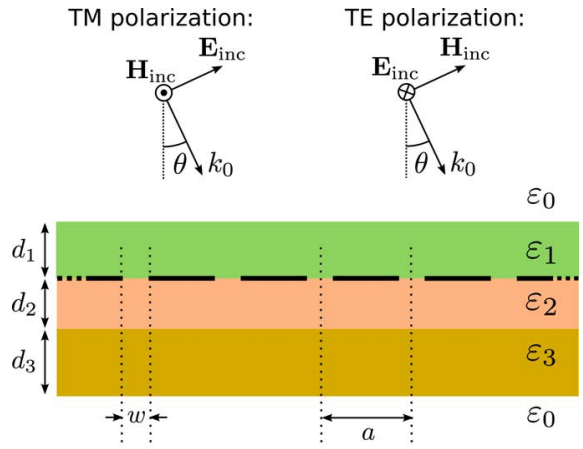

(a)

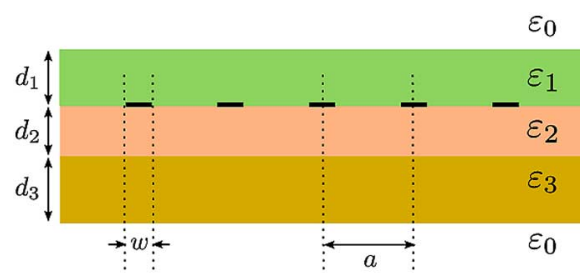

(b)

Fig. 1. Schematic representation of the problems under study. TM and TE polarizations and oblique incidence of the impinging wave are considered. (a) Slit-like 1-D planar grating with a stratified dielectric medium. (b) Strip-like $1-D$ planar grating in the same stratified environment.

[7]-[9], including infrared [10], [11] and terahertz applications [12], [13]. Apart from the technological interest of the practical applications of those structures, scientific curiosity has also triggered a lot of research on periodic planar or quasi-planar structures since the advent of extraordinary optical transmission [14]-[16].

The simplest geometry of the kind discussed above consists of an infinite 1-D periodic array of planar metal strips. As shown in Fig. 1, the strips could be embedded in a multilayered dielectric environment. In spite of its simplicity, this structure has been employed in different applications and frequency ranges, even in very recent studies [6], [9], [17]-[21]. Due to its geometrical simplicity, the structure can be analyzed with numerical methods allowing for a high degree of analytical preprocessing [22]. Under certain restrictions, it is possible to develop analytical or quasi-analytical methods, such as those found in [23]-[25]. Some of the authors of this paper have recently introduced an equivalent-circuit model involving lumped elements and transmission lines that removes many of the limitations encountered by previously developed analytical approaches [26], [27]. The same kind of model has also been used to explain an interesting phenomenon exhibited by strip-like structures under TE illumination [28], [29] 
(anomalous extraordinary transmission) or to design a terahertz polarizer [30]. The model is based on the idea of considering each unit cell of the periodic structure as a virtual parallel-plate waveguide discontinuity problem. This point of view is explicitly used in [31] and [32] to generate the numerical model and, to the authors' knowledge, the first wideband circuit model approach is fully developed in [33]. In that paper, a few parameters (such as the values of lumped capacitors and inductors) were numerically obtained from full-wave solutions at a reduced number of frequency points. The approach in [27] for strip-like structures loaded with dielectric slabs took a step further in the analytical path, but still needed the numerical computation of one lumped component. The formulation in [27] started with a simplified mode matching scheme where a number of reasonable approximations were included. Our first aim in this paper is to eliminate the need for any kind of numerical simulations and to extend the scope of the work previously presented in [34]. This is done by starting with an integral-equation formulation for the electric field along the slits or for the electric current supported by the metal strips. If a reasonable assumption for those quantities is done, the proposed procedure leads to a fully analytical model (readily cast in the form of an equivalent circuit), where all the parameters are known in closed form. In contrast with other intuitive circuit-like approaches, lumped capacitors (inductors) do not necessarily coincide with the electrostatic (magnetostatic) values since frequency-dependent information associated with the lowest order scattered TE/TM modes is explicitly extracted out. This procedure leads to a very wideband equivalent circuit that is valid from zero frequency up to frequencies above the onset of the first few grating lobes. This is true for any angle of incidence provided the slits or the strips are electrically narrow. Apart from its fully analytical nature, another significant advantage of the formulation presented in this paper (with respect to the most closely related previous development in [27] and [34]) lies on its ability to deal with conductor backed strip/slit-like structures or with two stacked identical gratings. The interaction between two closely spaced gratings or the interaction of a single grating with a ground plane is accurately incorporated to the model. This has been done by considering all the relevant modes involved in the interaction, and not only the fundamental mode as it has been commonly done (see, e.g., [35]). A number of examples will illustrate the accuracy and wideband behavior of the proposed analytical model. The analytical results will be compared with a highly accurate and numerically efficient integral-equation formulation using full domain basis functions that incorporate the actual physical edge behavior.

\section{Derivation of the Analytical Model}

The derivation of the equivalent-circuit models here proposed follows a rationale similar to [27], but is based on an integralequation approach instead of a mode-matching scheme. First we derive the circuit model for a single grating placed between two different dielectric half spaces and later the presence of dielectric layers (see Fig. 1) is incorporated in the model by cascading transmission line sections, each of which corresponds to
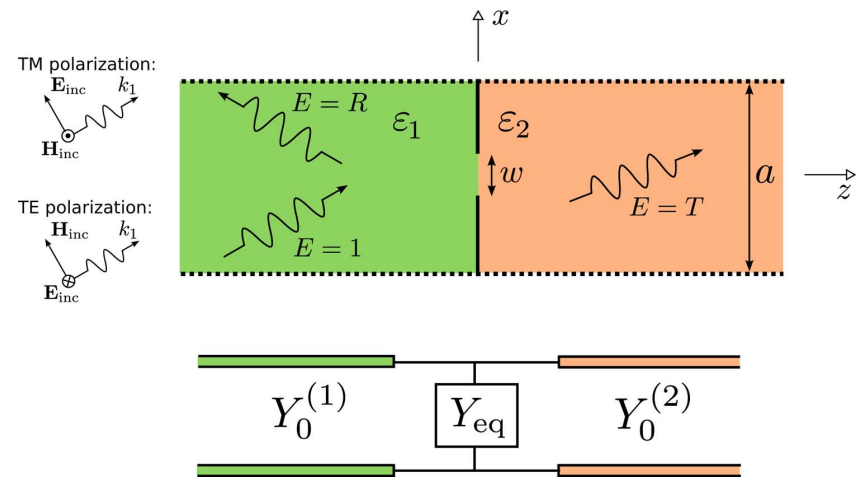

Fig. 2. (top) Unit cell corresponding to a periodic structure consisting of a periodic array of electrically narrow slits. (bottom) Circuit model to account for the scattering coefficients of the impinging electromagnetic wave.

a dielectric layer. Thus, if we consider the structure depicted in Fig. 2, and assume an implicit time-harmonic dependence of the type $\exp (-j \omega t)$, the transverse electric field at the screen $(z=0)$ can be expanded in a Floquet series of spatial harmonics as

$$
E(x)=(1+R) \mathrm{e}^{-\mathrm{j} k_{t} x}+\sum_{\substack{n=-\infty \\ n \neq 0}}^{\infty} E_{n} \mathrm{e}^{-\mathrm{j}\left(k_{t}+k_{n}\right) x}
$$

where the amplitude of the impinging wave has been normalized to unity, $R$ is the reflection coefficient of the zeroth harmonic, $k_{t}$ is the tangential-to-the-grating component of the wave vector of the obliquely incident plane wave (in the present case, $k_{t}=$ $k_{1} \sin \theta$, with $\left.k_{1}=\omega \sqrt{\varepsilon_{1} \mu_{0}}\right)$, and $k_{n}=2 \pi n / a$. In the following, we will suppress the range of the index $n$ in the summation if it is from $-\infty$ to $\infty$. Since the electric field is continuous across the dielectric interface and zero on the perfect conducting screen, the same expansion coefficients are valid at both sides of the grating, which implicitly implies $1+R=T$ with $T$ being the transmission coefficient. The transverse magnetic field at both sides of the grating $\left[H^{(1)}(x)=H\left(x, z=0^{-}\right)\right.$and $\left.H^{(2)}(x)=H\left(x, z=0^{+}\right)\right]$is then given by

$$
\begin{aligned}
& H^{(1)}(x)=Y_{0}^{(1)}(1-R) \mathrm{e}^{-\mathrm{j} k_{t} x}-\sum_{n \neq 0} Y_{n}^{(1)} E_{n} \mathrm{e}^{-\mathrm{j}\left(k_{t}+k_{n}\right) x} \\
& H^{(2)}(x)=Y_{0}^{(2)}(1+R) \mathrm{e}^{-\mathrm{j} k_{t} x}+\sum_{n \neq 0} Y_{n}^{(2)} E_{n} \mathrm{e}^{-\mathrm{j}\left(k_{t}+k_{n}\right) x}
\end{aligned}
$$

where

$$
Y_{n}^{(i)}= \begin{cases}\frac{\beta_{n}^{(i)}}{\omega \mu_{0}} & \text { TE harmonics } \\ \frac{\omega \varepsilon_{i}}{\beta_{n}^{(i)}} & \text { TM harmonics. }\end{cases}
$$

is the transverse (with respect to $z$ ) wave admittance of the $n$th harmonic in the $i$ th medium with

$$
\beta_{n}^{(i)}=\sqrt{\omega^{2} \varepsilon_{i} \mu_{0}-\left(k_{t}+k_{n}\right)^{2}}
$$

being its propagation wavenumber along $z$. Next we consider separately the cases of slit and strip gratings. 


\section{A. Slit Gratings}

If we denote the transverse electric field at the slit aperture as $E_{\mathrm{s}}(x)$, the coefficients of the electric field expansion in (1) can be obtained by standard Fourier analysis as

$$
\begin{aligned}
1+R & =\frac{1}{a} \int_{-w / 2}^{w / 2} E_{\mathrm{s}}(x) \mathrm{e}^{\mathrm{j} k_{t} x} \mathrm{~d} x=\frac{1}{a} \tilde{E}_{\mathrm{s}}\left(k_{t}\right) \\
E_{n} & =\frac{1}{a} \int_{-w / 2}^{w / 2} E_{\mathrm{s}}(x) \mathrm{e}^{\mathrm{j}\left(k_{t}+k_{n}\right) x} \mathrm{~d} x=\frac{1}{a} \tilde{E}_{\mathrm{s}}\left(k_{t}+k_{n}\right)
\end{aligned}
$$

where the tilde over the slit field denotes Fourier transform. From the above equations, we can write

$$
E_{n}=(1+R) \frac{\tilde{E}_{\mathrm{s}}\left(k_{t}+k_{n}\right)}{\tilde{E}_{\mathrm{s}}\left(k_{t}\right)}
$$

which relates the coefficient of every spatial harmonic with the reflection coefficient. Now the continuity of the magnetic field through the slit is projected over the electric field at the aperture to obtain

$$
\int_{-w / 2}^{w / 2} E_{\mathrm{s}}^{*}(x)\left[H^{(2)}(x)-H^{(1)}(x)\right] \mathrm{d} x=0
$$

where the asterisk indicates the complex conjugate. Introducing the expansions of the magnetic field in (2) and (3) into (9), we find the following expression for the reflection coefficient:

$$
R=\frac{Y_{0}^{(1)}-Y_{0}^{(2)}-Y_{\mathrm{eq}}}{Y_{0}^{(1)}+Y_{0}^{(2)}+Y_{\mathrm{eq}}}
$$

where

$$
Y_{\mathrm{eq}}=\sum_{n \neq 0}\left|\frac{\tilde{E}_{\mathrm{s}}\left(k_{t}+k_{n}\right)}{\tilde{E}_{\mathrm{s}}\left(k_{t}\right)}\right|^{2}\left(Y_{n}^{(1)}+Y_{n}^{(2)}\right)
$$

is identified as the global admittance of the equivalent circuit in Fig. 2. This admittance indicates that the equivalent circuit consists of a parallel connection of the wave admittances of the higher order spatial harmonics at both sides of the metallic grating, multiplied by a factor related to the spatial spectrum of the field profile at the slit (this factor could be interpreted as a transformer ratio). Unlike [27], these factors can now incorporate the a priori knowledge of the approximated field profile in the aperture (in [27] these factors were independent of $n$ as well as the incidence angle). Although (11) is a rigorous expression that is valid at any given frequency and for any slit width, in principle it is of little practical use unless the electric field at the slit aperture is known. Fortunately, for electrically narrow slits, we can make the reasonable assumption that the aperture field is given by

$$
E_{\mathrm{s}}(x)=A f(x)
$$

where $A$ is a frequency-dependent complex amplitude. This assumption implies that the spatial profile of the aperture field remains invariant in the frequency range of interest (in our experience, this condition is well satisfied up to the limit $w \lesssim 0.15 \lambda_{0}$; no specific restrictions are imposed on the period and the angle of incidence). Note that $A$ does not appear in the final expression of the equivalent circuit admittance (11) since this admittance only depends on the ratio of $\tilde{E}_{\mathrm{s}}\left(k_{t}+k_{n}\right)$ to $\tilde{E}_{\mathrm{s}}\left(k_{t}\right)$. It is interesting to note that this procedure to find the equivalent admittance could be viewed, after using (12) in (9), as a method-ofmoments solution (with a Galerkin testing scheme) of the magnetic field integral equation for the slit aperture when using one single basis function, $f(x)$, for the unknown magnetic current (electric field) at the slit.

For TM polarization, a suitable choice for the field profile is

$$
f(x) \propto\left[1-\left(\frac{2 x}{w}\right)^{2}\right]^{-1 / 2} \quad \tilde{f}(k) \propto J_{0}\left(\frac{k w}{2}\right)
$$

and for TE polarization,

$$
f(x) \propto\left[1-\left(\frac{2 x}{w}\right)^{2}\right]^{1 / 2} \quad \tilde{f}(k) \propto \frac{J_{1}\left(\frac{k w}{2}\right)}{\frac{k w}{2}}
$$

where $J_{n}(\cdot)$ represents the Bessel function of the first kind and order $n$. These field profiles incorporate the proper (singular or zero) behavior of the field at the slit edges in each case.

In the quasi-static limit, it is important to realize that the equivalent-circuit admittance in (11) can be conveniently approximated as

$$
Y_{\mathrm{eq}} \approx \sum_{n \neq 0}\left[\frac{\tilde{f}\left(k_{n}\right)}{\tilde{f}\left(k_{t}\right)}\right]^{2}\left(Y_{n, \mathrm{qs}}^{(1)}+Y_{n, \mathrm{qs}}^{(2)}\right)
$$

where it has been assumed that $k_{t} \ll k_{n}$. This allows us to write

$$
\begin{aligned}
k_{t} \pm k_{n} & \approx k_{n} \\
\beta_{n}^{(i)} & \approx-\mathrm{j}\left|k_{n}\right|
\end{aligned}
$$

and also [taking into account (4)]

$$
Y_{n, q \mathrm{qs}}^{(i)}= \begin{cases}\frac{-\mathrm{j}\left|k_{n}\right|}{\omega \mu_{0}}=\frac{1}{\frac{\mathrm{j} \omega \mu_{0}}{\left|k_{n}\right|}} & \text { TE harmonics } \\ \frac{\omega \varepsilon_{i}}{-\mathrm{j}\left|k_{n}\right|}=\frac{\mathrm{j} \omega \varepsilon_{i}}{\left|k_{n}\right|} & \text { TM harmonics. }\end{cases}
$$

The above derivation implies that the equivalent quasi-static admittance can be expressed in terms of a lumped inductance/capacitance (for TE/TM harmonics) given by the following frequency-independent series:

$$
\begin{aligned}
\frac{1}{L_{\mathrm{qs}}} & =\frac{4}{\mu_{0}\left[\tilde{f}\left(k_{t}\right)\right]^{2}} \sum_{n=1}^{\infty}\left[\tilde{f}\left(k_{n}\right)\right]^{2} k_{n} \\
C_{\mathrm{qS}} & =\frac{2\left(\varepsilon_{1}+\varepsilon_{2}\right)}{\left[\tilde{f}\left(k_{t}\right)\right]^{2}} \sum_{n=1}^{\infty} \frac{\left[\tilde{f}\left(k_{n}\right)\right]^{2}}{k_{n}} .
\end{aligned}
$$

Thus, (19) and (20) provide analytical expressions of the quasi-static inductance/capacitance of a narrow-slit grating under TE/TM incidence. These lumped quasi-static elements can be used in simple models for dense gratings where the period is much smaller than the wavelength (see, e.g., [5]). However, as frequency increases, it is clear that the contribution of the first harmonics to the equivalent admittance of the grating 


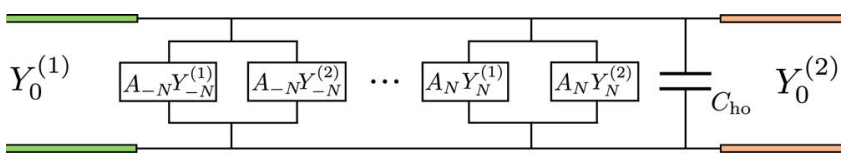

(a)

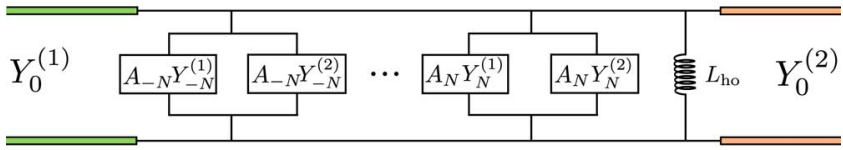

(b)

Fig. 3. Proposed equivalent network for the slit grating in Fig. 2. (a) TM polarization. (b) TE polarization.

starts to deviate from its quasi-static behavior. Therefore, in order to improve the above approximation so that it can be employed beyond the quasi-static limit, we propose to express the equivalent admittance as follows (see Fig. 3):

$$
\begin{array}{r}
Y_{\text {eq }} \approx \sum_{\substack{n=-N \\
n \neq 0}}^{N}\left[\frac{\tilde{f}\left(k_{t}+k_{n}\right)}{\tilde{f}\left(k_{t}\right)}\right]^{2}\left(Y_{n}^{(1)}+Y_{n}^{(2)}\right) \\
+ \begin{cases}j \omega C_{\mathrm{ho}} & \text { TM polarization } \\
\frac{1}{j \omega L_{\mathrm{ho}}} & \text { TE polarization. }\end{cases}
\end{array}
$$

The first series in (21) explicitly takes into account the frequency dependence of the dominant harmonics, whereas the last term in (21) is an admittance that incorporates the effect of all the scattered harmonics of order $|n|>N$ in the form of a lumped frequency-independent inductance/capacitance $\left(L_{\mathrm{ho}} / C_{\mathrm{ho}}\right)$. This "high-order" inductance/capcitance is given by the same series as in (19) and (20), but now the sum starts from $n=N+1$. In our experience, the approximation (21) provides sufficiently accurate values if $N$ is taken as 1 plus the number of propagative harmonics in the medium with highest permittivity. In most practical cases, it implies that $N$ is rarely greater than 3 or 4 .

The presence of dielectric slabs in the structure under study is taken into account by introducing the corresponding transmission-line sections. The wave admittances in (11) and elsewhere should then be replaced by the input admittances to the corresponding cascade of transmission lines seen from the grating to the left and right $\left(Y_{\mathrm{in}, n}^{\mathrm{L}}\right.$ and $\left.Y_{\mathrm{in}, n}^{\mathrm{R}}\right)$. In order to clarify this point, Fig. 4 shows an example of the circuit model for a printed slit grating under TM incidence $\left(\varepsilon_{1}=\varepsilon_{r} \varepsilon_{0}\right)$, with the abovementioned input admittances schematically represented at the bottom. The parameters of this equivalent network are given by

$$
\begin{aligned}
A_{n} & =\left[\frac{\tilde{f}\left(k_{t}+k_{n}\right)}{\tilde{f}\left(k_{t}\right)}\right]^{2} \\
Y_{\mathrm{in}, n}^{\mathrm{L}} & =Y_{n}^{(0)} \\
Y_{\mathrm{in}, n}^{\mathrm{R}} & =Y_{n}^{(1)} \frac{Y_{n}^{(0)}+\mathrm{j} Y_{n}^{(1)} \tan \left(\beta_{n}^{(1)} d\right)}{Y_{n}^{(1)}+\mathrm{j} Y_{n}^{(0)} \tan \left(\beta_{n}^{(1)} d\right)}
\end{aligned}
$$
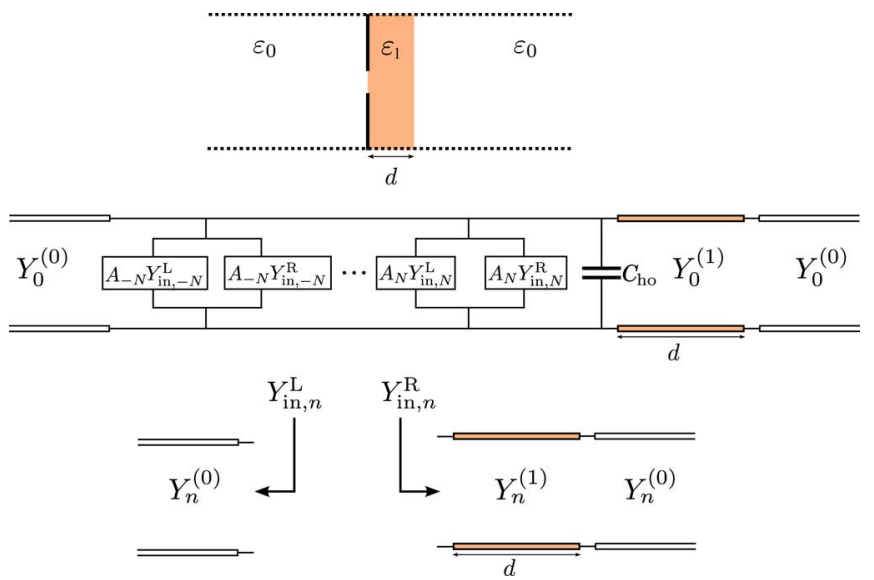

Fig. 4. Equivalent network for a TM wave impinging obliquely from the left $\left(k_{t}=k_{0} \sin \theta\right)$ on a printed slit grating, $\varepsilon_{1}=\varepsilon_{0} \varepsilon_{r}$.

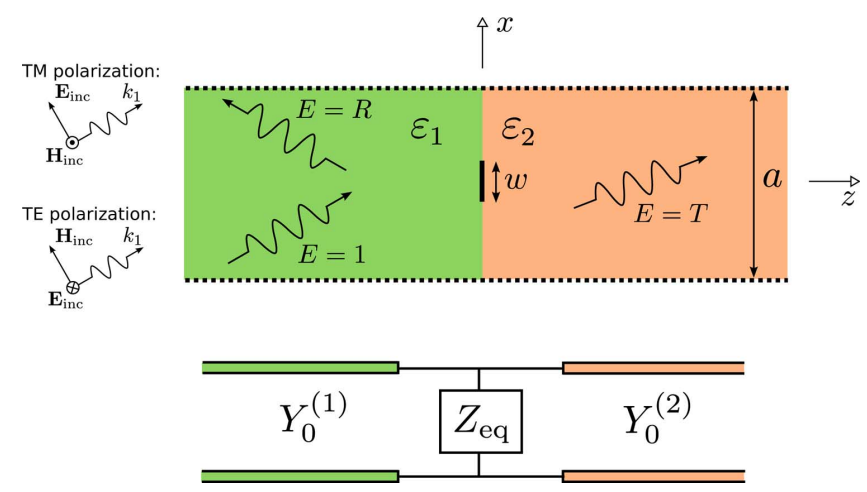

Fig. 5. Unit cell corresponding to a periodic structure consisting of a periodic array of electrically narrow strips.

$$
\begin{aligned}
C_{\mathrm{ho}}= & \frac{2 \varepsilon_{0}}{\left[\tilde{f}\left(k_{t}\right)\right]^{2}} \sum_{n=N+1}^{\infty} \frac{\left[\tilde{f}\left(k_{n}\right)\right]^{2}}{k_{n}} \\
& \times\left\{1+\varepsilon_{r} \frac{1+\varepsilon_{r} \tanh \left(k_{n} d\right)}{\varepsilon_{r}+\tanh \left(k_{n} d\right)}\right\} .
\end{aligned}
$$

Note that (24) is the resulting transformed admittance when the "load" admittance corresponding to the $n$th harmonic of the right-most transmission line, $Y_{n}^{(0)}$, is viewed through a length $d$ of transmission line characterized by $Y_{n}^{(1)}$ and $\beta_{n}^{(1)}$. The expression of the high-order capacitance in (25) can be readily obtained after introducing the approximations (16) and (17) in the expressions of the input admittances of the harmonics of order higher than $N$.

\section{B. Strip Gratings}

In the situation shown in Fig. 5, we start by considering the surface current on the strips, which is denoted as $J_{\mathrm{s}}(x)$. This surface current must equal the discontinuity of the transverse magnetic field, namely,

$$
H^{(1)}(x)-H^{(2)}(x)=J_{\mathrm{s}}(x) \text {. }
$$


Using the magnetic field expansions (2) and (3), the above expression can be written as

$$
\begin{aligned}
Y_{0}^{(1)}(1-R)-Y_{0}^{(2)}(1+R) & =\frac{1}{a} \tilde{J}_{\mathrm{s}}\left(k_{t}\right), \\
-Y_{n}^{(1)} E_{n}-Y_{n}^{(2)} E_{n} & =\frac{1}{a} \tilde{J}_{\mathrm{s}}\left(k_{t}+k_{n}\right)
\end{aligned}
$$

from which it is obtained that

$$
E_{n}=\frac{\tilde{J}_{\mathrm{s}}\left(k_{t}+k_{n}\right)}{\tilde{J}_{\mathrm{s}}\left(k_{t}\right)} \frac{R\left(Y_{0}^{(1)}+Y_{0}^{(2)}\right)-Y_{0}^{(1)}+Y_{0}^{(2)}}{Y_{n}^{(1)}+Y_{n}^{(2)}} .
$$

Using the electric field integral equation,

$$
\int_{-w / 2}^{w / 2} J_{\mathrm{s}}^{*}(x) E(x) \mathrm{d} x=0
$$

and after making the same assumption as in (12), but now for $J_{\mathrm{s}}(x)$, we finally find that

$$
\begin{array}{r}
Z_{\text {eq }} \approx \sum_{\substack{n=-N \\
n \neq 0}}^{N}\left[\frac{\tilde{f}\left(k_{t}+k_{n}\right)}{\tilde{f}\left(k_{t}\right)}\right]^{2} \frac{1}{Y_{n}^{(1)}+Y_{n}^{(2)}} \\
+ \begin{cases}\mathrm{j} \omega L_{\mathrm{ho}} & \text { TE polarization } \\
\frac{1}{\mathrm{j} \omega C_{\mathrm{ho}}} & \text { TM polarization. }\end{cases}
\end{array}
$$

where the high-order inductance and capacitance are now given by

$$
\begin{aligned}
L_{\mathrm{ho}} & =\frac{\mu_{0}}{\left[\tilde{f}\left(k_{t}\right)\right]^{2}} \sum_{n=N+1}^{\infty} \frac{\left[\tilde{f}\left(k_{n}\right)\right]^{2}}{k_{n}} \\
\frac{1}{C_{\mathrm{ho}}} & =\frac{2}{\left(\varepsilon_{1}+\varepsilon_{2}\right)\left[\tilde{f}\left(k_{t}\right)\right]^{2}} \sum_{n=N+1}^{\infty}\left[\tilde{f}\left(k_{n}\right)\right]^{2} k_{n} .
\end{aligned}
$$

It should be noted that now the surface current profile $f(x)$ is given by (14) for TM polarization and (13) for TE polarization of the impinging wave.

The inclusion of dielectric layers can be done in a parallel way as that explained for the slit case. As an example, we can consider the case of a TM wave impinging on a printed strip shown in Fig. $6\left(\varepsilon_{1}=\varepsilon_{0} \varepsilon_{r}\right)$. The equivalent network is also shown in the figure. The network parameters $A_{n}, Y_{\mathrm{in}, n}^{\mathrm{L}}$, and $Y_{\mathrm{in}, n}^{\mathrm{R}}$ are given by the same expressions in (22)-(24), respectively, although with appropriate profile $f(\cdot)$. The high-order capacitance is given by

$$
\begin{aligned}
\frac{1}{C_{\mathrm{ho}}}=\frac{2}{\varepsilon_{0}\left[\tilde{f}\left(k_{t}\right)\right]^{2}} & \sum_{n=N+1}^{\infty}\left[\tilde{f}\left(k_{n}\right)\right]^{2} k_{n} \\
& \times\left\{1+\varepsilon_{r} \frac{1+\varepsilon_{r} \tanh \left(k_{n} d\right)}{\varepsilon_{r}+\tanh \left(k_{n} d\right)}\right\}^{-1} .
\end{aligned}
$$

\section{Model For Coupled GRATINGS}

In this section, we extend the previous circuit models to study the case of symmetric coupled gratings. The structures under consideration are sketched in Fig. 7, which also illustrates the

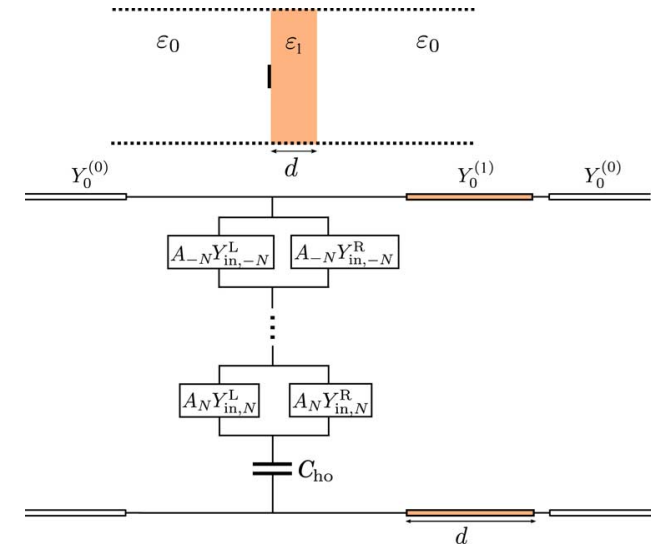

Fig. 6. Equivalent network for a TM wave impinging obliquely from the left $\left(k_{t}=k_{0} \sin \theta\right)$ on a printed strip grating, $\varepsilon_{1}=\varepsilon_{0} \varepsilon_{r}$.

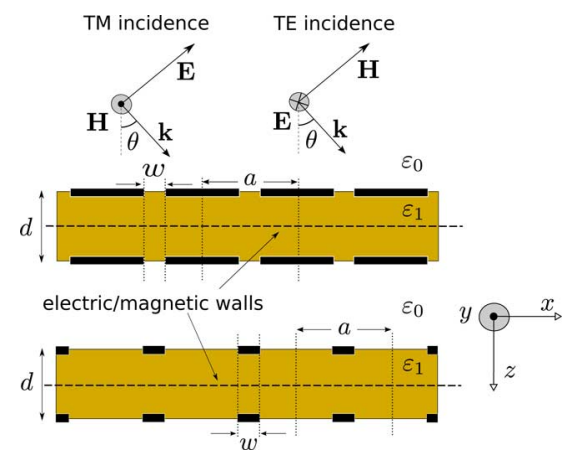

Fig. 7. Schematic representation of the coupled slit and strip gratings under analysis. The TE and TM oblique incidences are also represented on top. The optional electric wall condition at the middle plane accounts for the grating printed on a grounded slab, and also corresponds to the odd excitation of the coupled slit/strip gratings. The magnetic wall condition corresponds to the even excitation.

two cases of TE and TM oblique incidence. The structure shown at the top represents a pair of 1-D periodic array of slits made in an infinitely thin metallic screen printed on both sides of a dielectric slab (this structure can also be seen as a pair of coupled wide-strip gratings). The quasi-complementary problem involving strip gratings is shown at the bottom. The structures under study show a symmetry plane at the middle of the slab. This symmetry plane allows us to decompose the impinging excitation as the superposition of even (e) plus odd (o) excitations. Under even/odd excitation, this symmetry plane behaves as a magnetic/electric wall. The scattering parameters of the coupled structures can readily be found from the scattering parameters of the structures with even/odd excitations as

$$
\begin{aligned}
S_{11} & =\frac{1}{2}\left(S_{11}^{\mathrm{e}}+S_{11}^{\mathrm{o}}\right) \\
S_{21} & =\frac{1}{2}\left(S_{11}^{\mathrm{e}}-S_{11}^{\mathrm{o}}\right) .
\end{aligned}
$$

The case of a grating printed on a grounded slab is implicitly considered in this analysis since it is equivalent to the electric wall boundary condition at the middle plane of the dielectric slab.

Next we present in some detail the derivation of the circuit model for the particular case of a strip grating printed on a grounded dielectric slab under TE incidence. The derivation for 


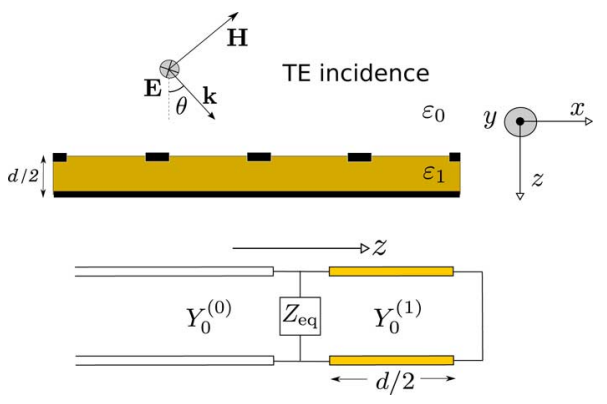

Fig. 8. Equivalent network to model the slit grating printed on a grounded slab under TE polarization. The short-circuit termination accounts for the presence of the ground plane. The shunt impedance $Z_{\text {eq }}$ is the global impedance of the equivalent circuit that models the effect of the periodic grating.

TM incidence and for the slit grating under TE/TM incidence can be carried out along similar lines and will not be explicitly shown. Thus, we consider the strip grating in Fig. 7 with the electric wall condition (ground plane) at the middle. Following the approach discussed in Section II, the equivalent-circuit model of this structure is that depicted in Fig. 8. The transmission lines to the left and right of $Z_{\text {eq }}$ account for the propagation of the zeroth-order harmonic (incident and reflected wave) in free space and inside the dielectric, respectively. The short-circuit termination accounts for the presence of the ground plane, and the shunt impedance $Z_{\text {eq }}$ represents the global impedance of the equivalent circuit that models the periodic screen, which is given by

$$
\begin{array}{r}
Z_{\text {eq }} \approx \sum_{\substack{n=-N \\
n \neq 0}}^{N}\left[\frac{\tilde{f}\left(k_{t}+k_{n}\right)}{\tilde{f}\left(k_{t}\right)}\right]^{2} \frac{1}{Y_{n}^{(0)}+Y_{\mathrm{in}, n}^{\mathrm{R}}} \\
+ \begin{cases}\mathrm{j} \omega L_{\mathrm{ho}} & \text { TE polarization } \\
\frac{1}{\mathrm{j} \omega C_{\mathrm{ho}}} & \text { TM polarization }\end{cases}
\end{array}
$$

where

$$
Y_{\mathrm{in}, n}^{\mathrm{R}}=-\mathrm{j} Y_{n}^{(1)} \cot \left(\beta_{n}^{(1)} \frac{d}{2}\right)
$$

In the particular case that $N=1$ and normal TE incidence, we find that the equivalent circuit reduces to that shown in Fig. 9, where it has been taken into account that $k_{t}=0$, and therefore, the admittances of the $n=1$ and $n=-1$ harmonics are identical and can be included in a single series element. The $A_{1}$ coefficient in Fig. 9 corresponds to (22) with $k_{t}=0$, and the $L_{\mathrm{ho}}$ inductance is given by

$$
L_{\mathrm{ho}}=\frac{2 \mu_{0}}{\tilde{f}^{2}(0)} \sum_{n=2}^{\infty} \frac{\tilde{f}^{2}\left(k_{n}\right)}{k_{n}}\left[1+\operatorname{coth}\left(\frac{k_{n} d}{2}\right)\right]^{-1} .
$$

For the case of a magnetic wall boundary condition, the derivation is completely analogous, but an open circuit should be considered instead of the short circuit in Fig. 8, and therefore, $\cot (\cdot)$ should be replaced with $-\tan (\cdot)$ in (38). Also, in the particular case of the inductance in $(39), \operatorname{coth}(\cdot)$ should be replaced with $\tanh (\cdot)$.

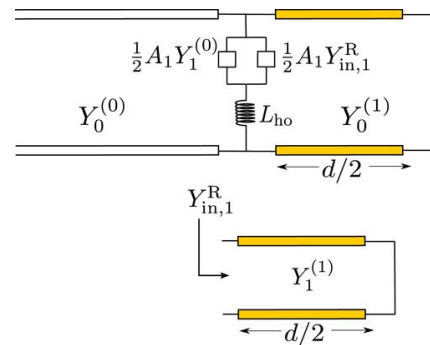

Fig. 9. Equivalent network for a strip grating printed on a grounded slab under normal TE incidence. Only the first element is explicitly taken into account with its complete frequency dependence $(N=1)$.

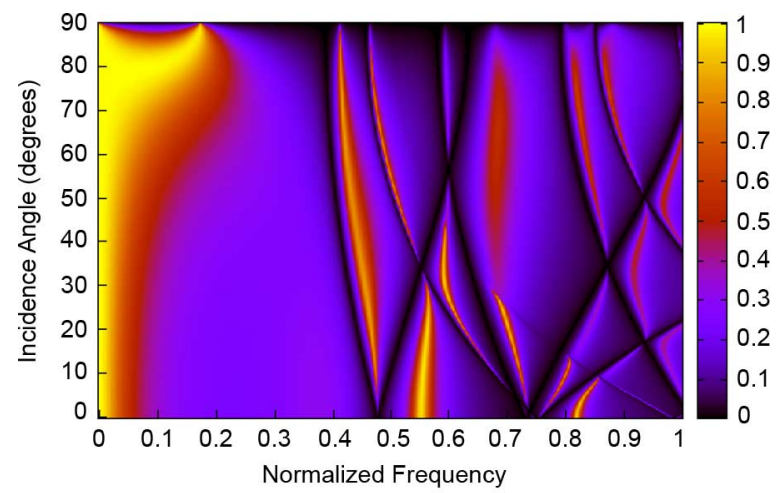

(a)

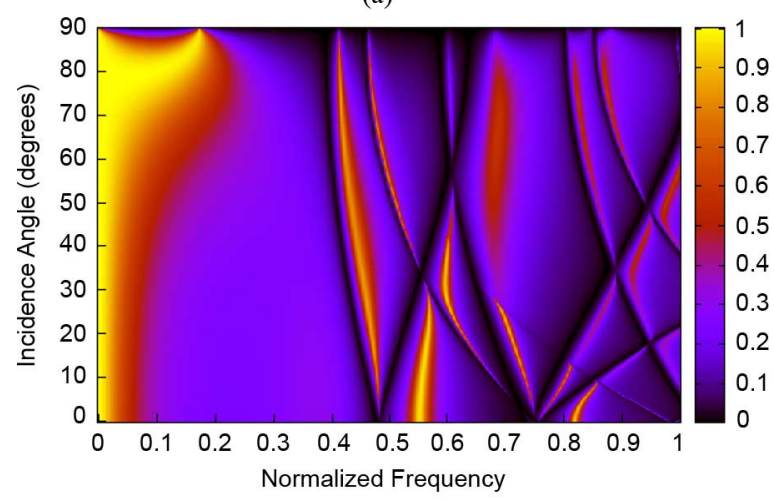

(b)

Fig. 10. Magnitude of the transmission coefficient under TM oblique incidence of a slit grating $(w=0.1 a)$ embedded between two dielectric layers with $\varepsilon_{1}=$ $2.2 \varepsilon_{0}, \varepsilon_{2}=10.2 \varepsilon_{0}, d_{1}=0.4 a, d_{2}=0.2 a$. Both dielectric layers are lossy with $\tan \delta=0.001$. (a) Circuit model results. (b) MoM results. Normalized frequency $a / \lambda_{0}$.

\section{NumERICAL RESUlTS AND Discussion}

The analytical model proposed in this paper has been developed on the basis of a certain number of assumptions concerning the width of slits (or strips) and the field distribution (current distribution) on those slits (strips). Moreover, some decisions have to be taken before generating numerical results concerning the number of TE/TM harmonics of relatively low order that must be explicitly retained in the formulation (the information of the remaining infinite higher order modes is summarized by the lumped elements). However, these issues have been treated in detail in our previous paper on the same topic [27]. One of the main differences between this paper and [27] lies in the development of a fully analytical circuit-like model 


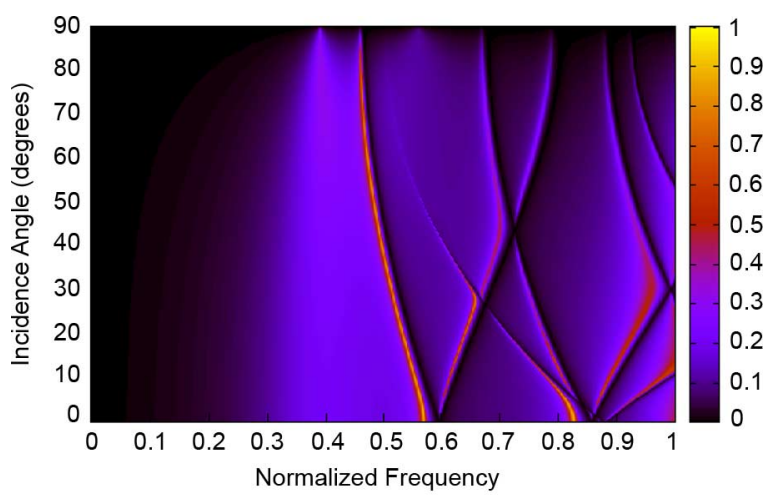

(a)

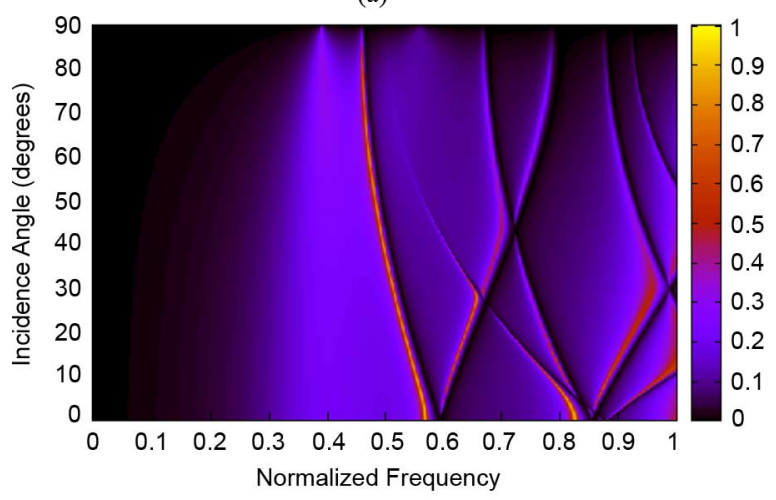

(b)

Fig. 11. Magnitude of the transmission coefficient under TE oblique incidence of a slit grating $(w=0.2 a)$ embedded between two dielectric layers with $\varepsilon_{1}=2.2 \varepsilon_{0}, \varepsilon_{2}=10.2 \varepsilon_{0}, d_{1}=0.4 a$, and $d_{2}=0.2 a$. Both dielectric layers are lossy with $\tan \delta=0.001$. (a) Circuit model results. (b) MoM results. Normalized frequency: $a / \lambda_{0}$.

(the lumped parameters in [27] were extracted from a numerical calculation for a single frequency point). In this paper, we have provided analytical formulas for all the relevant parameters, and it is necessary to check the accuracy of such formulas. However, the physical considerations about the number of modes that have to be retained to account for the (possibly) complex frequency-dependent behavior of the discontinuities are exactly the same as in [27]. The criterion here is to retain all the TE/TM harmonics that are above cutoff inside the highest dielectric constant slab plus the first evanescent higher order mode. Since the cutoff frequencies of the TE/TM harmonics are known in advance, this task can readily be implemented in the computer code. It should be noted that the relative weights of the modes calculated in [27] and in this paper are slightly different, with the ones in this paper being more accurate. As a validation of our present approach, we have recalculated with the new analytical formulation all the results already reported in [27] with an excellent agreement (these comparisons will not be explicitly shown). For further validation purposes, our results have also been compared with numerically generated data. A method of moments (MoM) in the spectral domain has been used to solve for the electric-field/surface-current integral equations for the slits/strips arrays. A sufficient number of entire-domain quasi-orthogonal basis functions that include the edge singularities have been employed to ensure high accuracy and fast convergence. The numerical results thus obtained

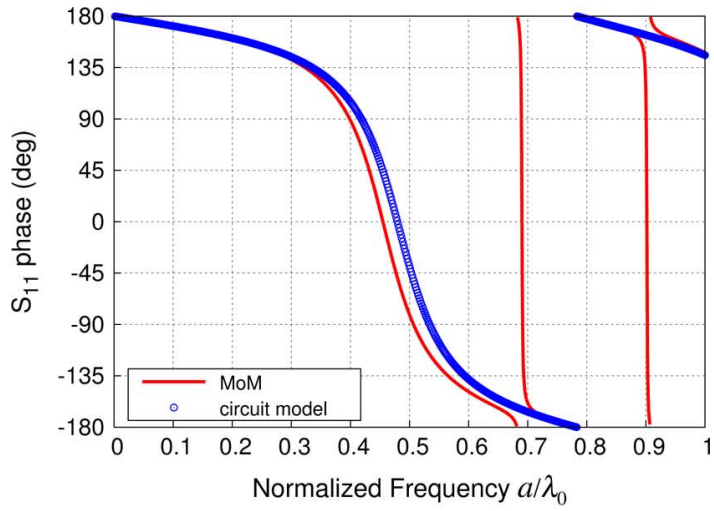

(a)

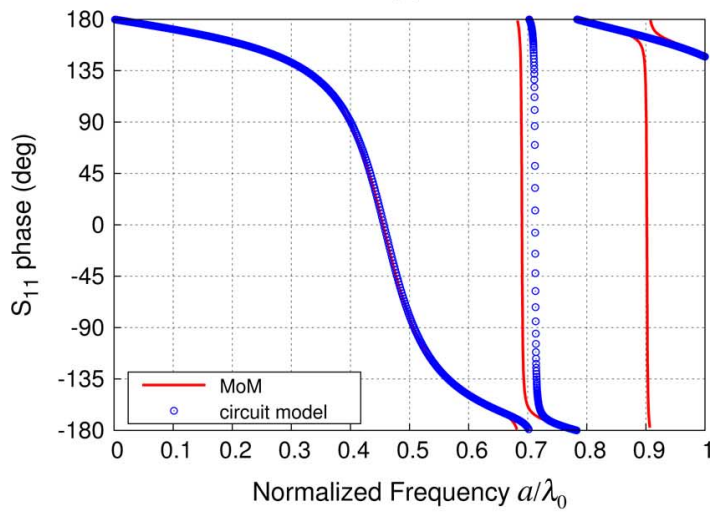

(b)

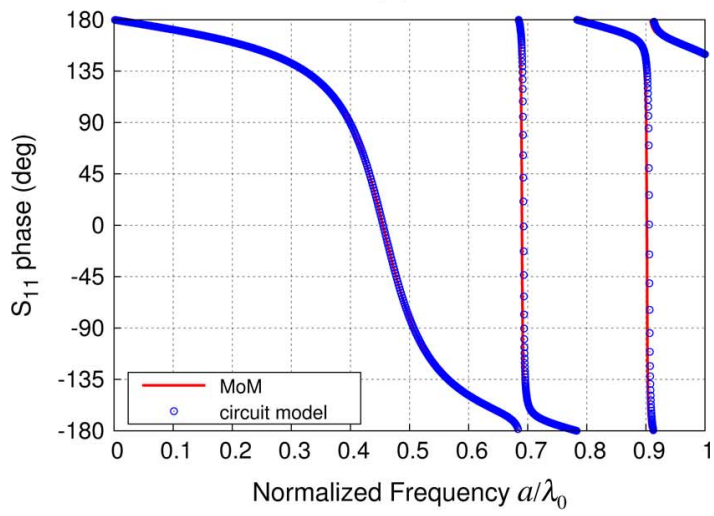

(c)

Fig. 12. Phase of the reflection coefficient for a strip grating printed on a lossless grounded slab under normal TE incidence. Structure parameters: $w=$ $0.1 a, \varepsilon_{r}=10.2, d / 2=0.2 a$. (a) $N=0$. (b) $N=1$. (c) $N=2$.

for the scattering coefficients are accurate within 4-5 significant figures at least. Differences between analytical and numerical data cannot be appreciated in the plots provided the width of the slits/strips is small in comparison with the wavelength (roughly $w / \lambda_{0}<0.15$ ). A very relevant fact is that the analytical model perfectly captures all the details of the transmission/reflection spectra: no peaks/dips are lost even for extremely complicated spectra exhibiting a large number of peaks/dips. In contrast with a model based on the excitation of surface waves [16], not only the frequency position of the peaks is almost exactly reproduced, but also the amplitude and phase at the peaks and at any other frequency over a very wide band. 


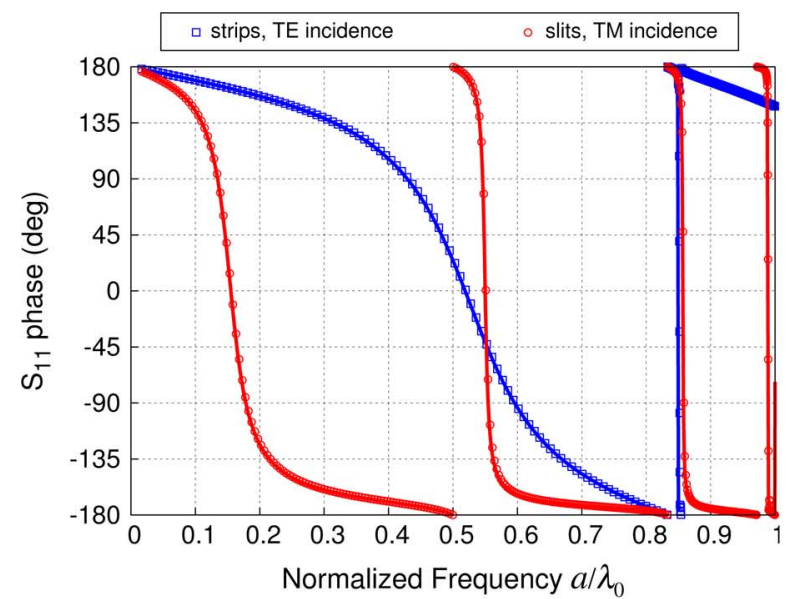

(a)

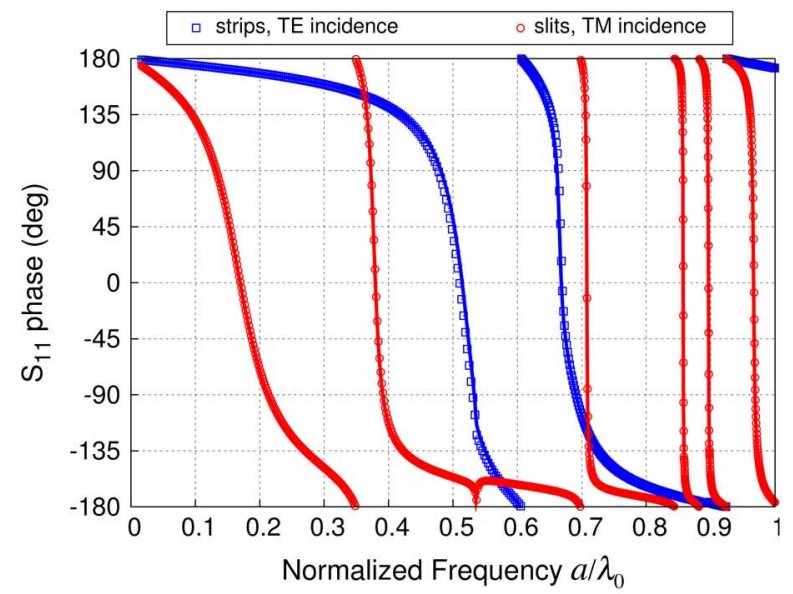

(c)

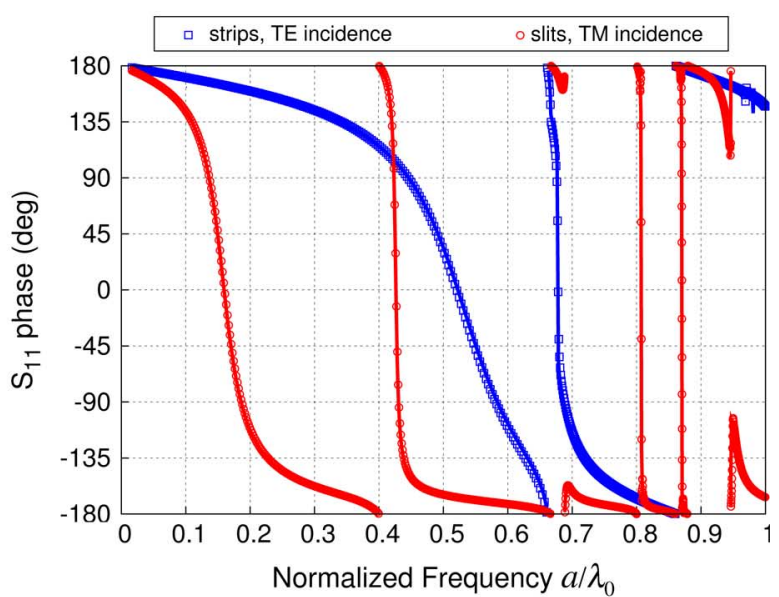

(b)

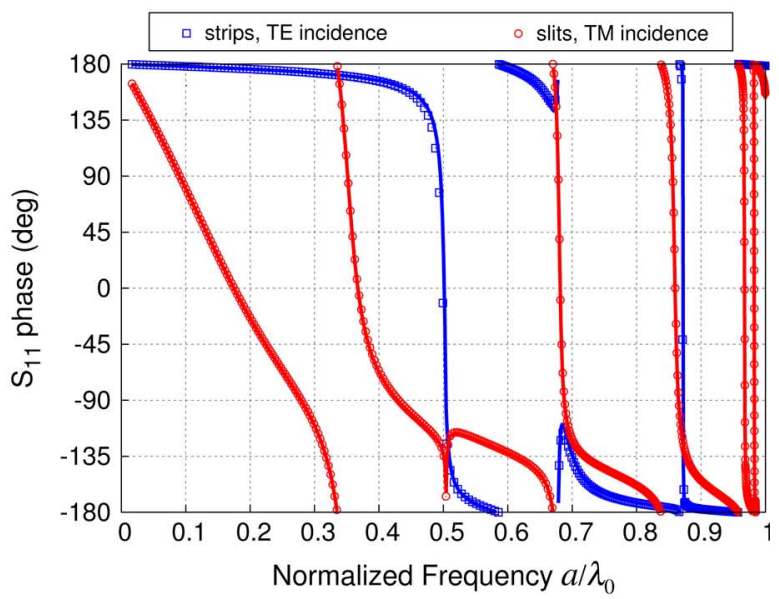

(d)

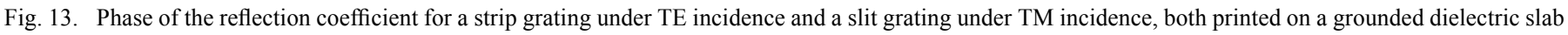

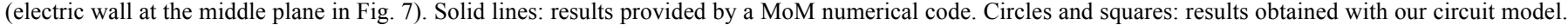

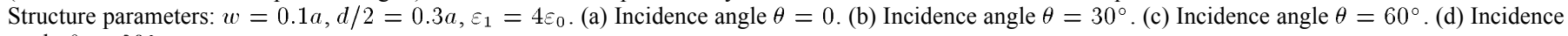
angle $\theta=80^{\circ}$.

In order to have a global idea about the performance of the model (although more detailed evidences can be found in [27]), we have included in Figs. 10 and 11 several illustrative color maps. These maps show the magnitude of the transmission coefficient for a couple of example structures as a function of the normalized frequency $\left(a / \lambda_{0}\right)$ and the angle of incidence. Fig. 10(a) has been generated with the analytical model in this paper, and corresponds to TM wave incidence on a grating of narrow slits sandwiched between two different slightly lossy dielectric slabs. The numerically generated (MoM) results have been plotted in Figs. 10(b). The two plots are almost identical. The spectrum for high frequencies $\left(a / \lambda_{0}>0.4\right)$ is very complex due to the presence of the dielectric slabs. Note that these kinds of plots are closely related to the Brillouin dispersion curves of the quasi-bound modes supported by the dielectric slabs periodically loaded with metal strips. It is worth mentioning that the explanation of the transmission peaks and zeros has been given, especially within the optics community, in terms of the interaction of the impinging TEM wave with the aforementioned quasibound modes (these modes were called spoof surface plasmons [36] due to their resemblance with the genuine surface plasmons supported by dielectric/solid plasma interfaces). The computa- tion of these Brillouin dispersion curves is usually carried out by numerically solving an eigenvalue problem that demands a lot of computational effort. In contrast, the plot in Fig. 10(a) was generated in seconds thanks to the analytical nature of the model. Similar plots for a TE illuminated slit-like planar metal grating are shown in Fig. 11. A wider slit is considered here in order to enhance the high transmission frequency regions. Once again, the agreement between the numerical results and the analytical data is very good. In these figures, a narrow high transmission peak in the frequency range going from $a / \lambda_{0} \approx 0.47$ to $a / \lambda_{0} \approx 0.6$ (depending on the angle of incidence) can be clearly appreciated. Immediately after this peak, a transmission zero appears (Fano-like resonance). This is the so-called anomalous extraordinary transmission [28], [29]. For this polarization and grating geometry, low transmission regions rather than transmission peaks should be expected in the absence of electrically thick dielectric layers. It is the presence of dense dielectric slabs what introduces the possibility of having transmission peaks in an otherwise mostly opaque screen. The qualitative explanation for this phenomenon was discussed in [27].

Previously we have considered structures similar to those studied in [27] with the difference of using the new analytical 


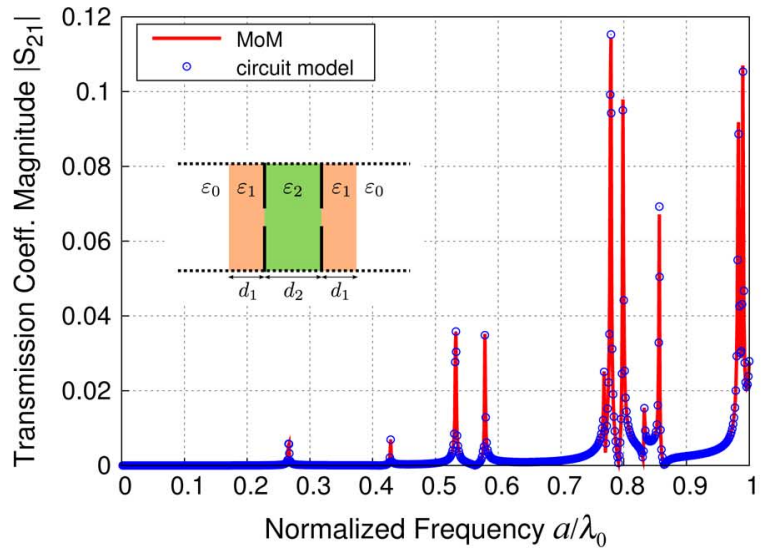

(a)

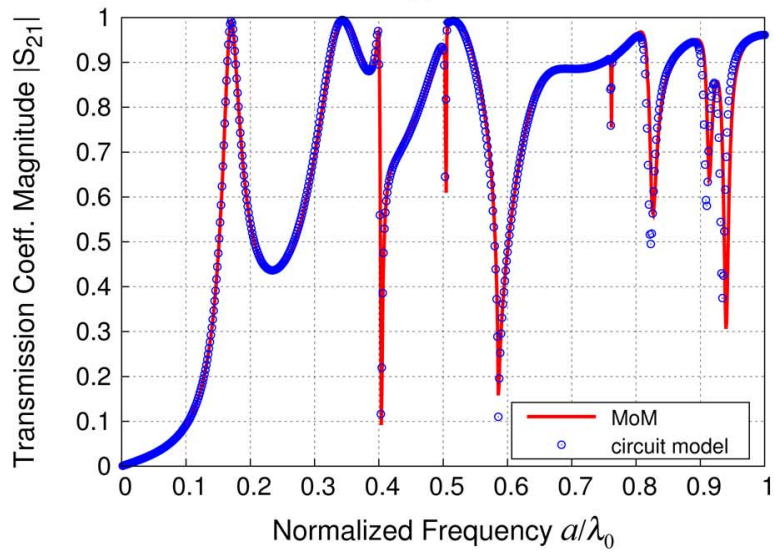

(c)

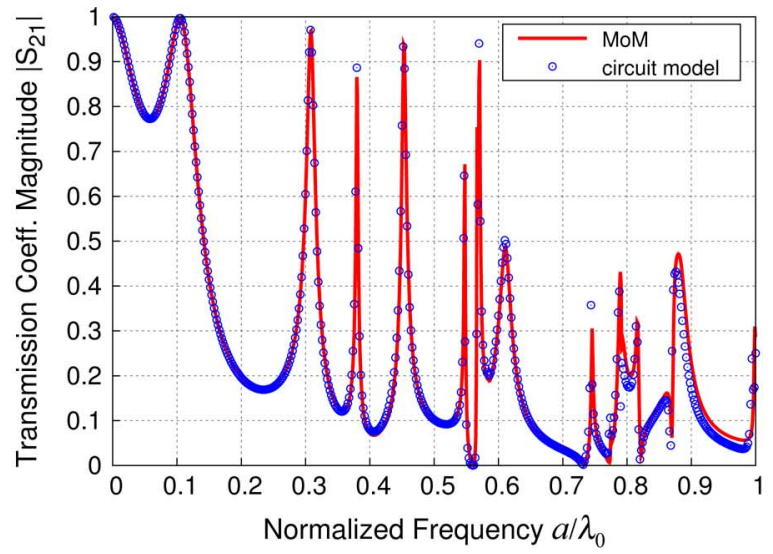

(b)

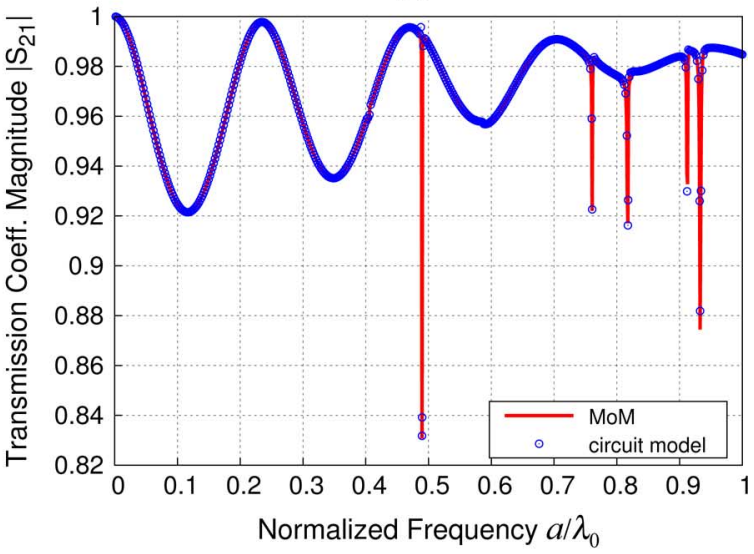

(d)

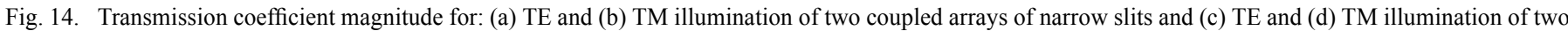

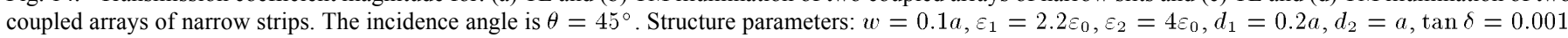
for all the layers.

tool. Now we consider conductor-backed or double-side structures for which no model was reported in [27]. This kind of structure can be studied in an approximate manner by using a lumped circuit component to account for the grating capacitance (TM case) or inductance (TE case) and a transmissionline section (with a short-circuit or an open-circuit termination) representing the grounded dielectric slab or the dielectric slab with a magnetic wall (symmetrical double-sided structures under even excitation conditions). This approach can be found, for instance, in [5] and [35]. Note that this transmission-line model only accounts for the fundamental TEM-like mode propagating inside the dielectric slab, and moreover, it does not consider the influence of the ground plane on the value of the lumped component. Thus, this representation ignores the possibility of higher order mode interaction of the grating with the ground plane (conductor-backed geometries) or with one adjacent identical grating (two-sided structures). At low frequencies, the higher order mode interaction can be ignored in practice if the dielectric slab is relatively thick or the value of $w$ is extremely small. However, significant differences between the analytical model and exact numerical calculations can be found in other cases. Furthermore, if the operation frequency is close or higher than the cutoff frequency of the first high-order harmonic inside the dielectric slab, then the coupling through this harmonic can strongly change the response of the structure. Certainly, this effect cannot be taken into account by simple quasi-static models. As was explained earlier, our model incorporates all the interactions by splitting the discontinuity (grating) influence into two contributions: the frequency-dependent contribution linked to the scattered low order TE/TM modes and the frequency-independent contribution of the high-order modes. If we make $N=0$ in our formulation, our model reduces to a quasi-static model similar to the above mentioned simpler approach [5], [35]. Actually, with $N=0$, we still keep the influence of the ground plane (or adjacent grating) on the quasi-static parameters characterizing the discontinuity, but this cannot account for the coupling through propagative harmonics at higher frequencies. To clarify this point, we compare the results obtained for an specific structure (strip grating printed on a grounded lossless slab) under normal TE illumination for several values of $N$. Since the amplitude of the reflection coefficient is unity, we only plot the phase of the reflection coefficient in Fig. 12. Although the quasi-static $(N=0)$ model gives accurate results up to $a / \lambda_{0}=0.3$, noticeable differences can be appreciated above that frequency. Indeed, the results provided by the quasi-static model are qualitatively incorrect for $a / \lambda_{0}>0.6$. However, if the first two relevant higher order modes are explicitly taken into account (i.e., they are not considered as part of the higher order lumped contribution), the analytical model perfectly captures the details of the phase be- 
havior up to the frequency of the onset of the first grating lobe. The model is still valid above that frequency but, in such a case, the magnitude of the specular reflection coefficient is not unity (depending on the operation frequency, parameter $N$ could need to be increased).

Next, Fig. 13 shows the phase of the reflection coefficient versus normalized frequency for strip/slit gratings printed on a grounded dielectric slab (see the caption for the structure parameters). The MoM numerical results are also shown, and the comparison with the analytical results shows an excellent agreement for all the cases. It is well known that, as frequency increases, these structures present a near-zero phase for the reflection coefficient (artificial magnetic conductor) in a given frequency range. In the normal incidence cases considered in Fig. 13(a), this behavior occurs around $a / \lambda_{0} \approx 0.52$ for the strip grating under TE illumination and around $a / \lambda_{0} \approx 0.16$ for the slit grating under TM illumination. These values remain approximately the same for the oblique incidence results shown in Figs. 13(b)-13(d). For the high-frequency portion of the spectrum, the phase exhibits a faster and more complicated variation with frequency, especially for oblique incidence.

As a final example, we have computed the transmission coefficient through a pair of symmetric coupled gratings printed on a thick substrate and sandwiched between two thinner dielectric slabs. The analytical and numerical results are compared in Fig. 14 for four different situations. The plots in Fig. 14(a) and (b) correspond to a pair of coupled slit-like (narrow slits) gratings under oblique $\left(\theta=45^{\circ}\right)$ TE and TM illuminations. The plots in Fig. 14(c) and (d) correspond to a pair of coupled strip-like (narrow strips) gratings under TE and TM illuminations. We can observe a very good agreement between numerical and analytical results in spite of the complexity of the transmission spectra. It is worth mentioning that simple circuit models that substitute the central dielectric slab with a TEM transmission line cannot be used when the operation frequency is close or above the cutoff frequencies of some of the first higher order TE or TM harmonics in the dielectric region. However, this is not an issue for our procedure. Finally, we should mention that, for wider strips, the results are slightly worse in the high-frequency part of the spectrum, but still acceptable for most practical purposes.

\section{CONClusions}

We have reported a fully analytical method to compute the transmission and reflection characteristics of strip- and slit-like diffraction gratings over a very wide frequency band (provided the strip/slit width is electrically small). The topology of the equivalent-circuit model and the values of their parameters (lumped capacitors/inductors and transmission lines) are extracted from a rigorous integral-equation formulation provided some reasonable approximations are employed. The proposed equivalent-circuit model can be seen as an alternative form of the integral-equation problem with the advantage that it provides more physical insight than the direct mathematical equations. Although other circuit-like models are available in the literature, the model here proposed systematically takes into account dynamic effects that are not usually accounted for. These effects are relevant at high frequencies, as well as when the metal gratings are close to a ground plane or strongly coupled to another identical grating, cases that have been specifically considered in this paper. The proposed model is useful to accurately characterize this kind of structures in classical microwave/millimeter-wave applications and to explain exotic phenomena recently reported in the optics and terahertz domains.

\section{REFERENCES}

[1] B. A. Munk, Frequency Selective Surfaces: Theory and Design. New York: Wiley, 2000.

[2] B. Gimeno, J. L. Cruz, E. A. Navarro, and V. Such, "A polarizer rotator system for three-dimensional oblique incidence," IEEE Trans. Antennas Propag., vol. 42, no. 7, pp. 912-919, Jul. 1994.

[3] G. F. Brand, "The strip grating as a circular polarizer," Amer. J. Phys., vol. 71, pp. 452-456, 2003 .

[4] M. A. Hiranandani, A. B. Yakovlev, and A. A. Kishk, "Artificial magnetic conductors realised by frequency-selective surfaces on a grounded dielectric slab for antenna applications," Proc. Int. Elect. Eng.-Microw., Antennas, Propag., vol. 153, no. 5, pp. 487-493, Oct. 2006

[5] O. Luukkonen, C. Simovski, G. Granet, G. Goussetis, D. Lioubtchenko, A. V. Risnen, and S. A. Tretyakov, "Simple and accurate analytical model of planar grids and high-impedance surfaces comprising metal strips or patches," IEEE Trans. Antennas Propag., vol. 56, no. 6, pp. 1624-1632, Jun. 2008.

[6] A. Foroozesh and L. Shafai, "On the characteristics of the highly directive resonant cavity antenna having metal strip grating superstrate," IEEE Trans. Antennas Propag., vol. 60, no. 1, pp. 78-91, Jan. 2012.

[7] G. Dolling, M. Wegener, C. M. Soukoulis, and S. Linden, "Negativeindex metamaterial at $780 \mathrm{~nm}$ wavelength," Opt. Lett., vol. 32, pp. 53-55, 2007.

[8] J. Zhang, W. Bai, L. Cai, Y. Xu, G. Song, and Q. Gan, "Observation of ultra-narrow band plasmon induced transparency based on large-area hybrid plasmon-waveguide systems," Appl. Phys. Lett., vol. 99, 2011, Art. ID 181120.

[9] P. Patoka, T. Sun, M. Giersig, Z. Ren, and K. Kempa, "Nanoribbon plasmonic gratings and their anomalous interaction with electromagnetic waves," Adv. Mater., vol. 24, pp. 3042-3045, 2012.

[10] R. Ulrich, "Far-infrared properties of metallic mesh and its complementary structure," Infrared Phys., vol. 7, pp. 37-55, 1967.

[11] R. Ulrich, "Effective low-pass filters for far infrared frequencies," Infrared Phys., vol. 7, pp. 65-74, 1967.

[12] M. E. MacDonald, A. Alexanian, R. A. York, Z. Popović, and E. N. Grossman, "Spectral transmittance of lossy printed resonant-grid terahertz bandpass filters," IEEE Trans. Microw. Theory Techn., vol. 48, no. 4, pp. 712-718, Apr. 2000.

[13] J. Carbonell, C. Croenne, F. Garet, E. Lheurette, J. L. Coutaz, and D. Lippens, "Lumped elements circuit of terahertz fishnet-like arrays with composite dispersion," J. Appl. Phys., vol. 108, 2010, Art. ID 014907.

[14] T. W. Ebbesen, H. J. Lezec, H. F. Ghaemi, T. Thio, and P. A. Wolff, "Extraordinary optical transmission through sub-wavelength hole arrays," Nature, vol. 391, pp. 667-669, Feb. 1998.

[15] F. J. García-de-Abajo, "Colloquium: Light scattering by particle and hole arrays," Rev. Mod. Phys., vol. 79, pp. 1267-1290, Oct.-Dec. 2007.

[16] F. J. García-Vidal, L. Martín-Moreno, T. W. Ebbesen, and L. Kuipers, "Light passing through subwavelength apertures," Rev. Mod. Phys., vol. 82, pp. 729-787, Jan.-Mar. 2010.

[17] E. Sakat, G. Vincent, P. Ghenuche, N. Bardou, C. Dupuis, S. Collin, F. Pardo, R. Haidar, and J.-L. Pelouard, "Free-standing guided-mode resonance and-pass filters: From 1-D to 2-D structures," Opt. Exp., vol. 20, no. 12, pp. 13082-13090, Jun. 2012.

[18] A. Khavasi, M. Miri, and K. Mehrany, "Surface plasmon-enhanced absorption in metal grating coupled terahertz quantum well photodetectors," IEEE Trans. Terahertz Sci. Technol., vol. 1, no. 2, pp. 435-440, Nov. 2011

[19] X. Guo, R. Zhang, J. Cao, and H. Liu, "Surface plasmon-enhanced absorption in metal grating coupled terahertz quantum well photodetectors," IEEE J. Quantum Electron., vol. 48, no. 9, pp. 1113-1119, Sep. 2012.

[20] J. L. Perchec, Y. Desieres, N. Rochat, and R. E. Lamaestre, "Subwavelength optical absorber with an integrated photon sorter," Appl. Phys. Lett., vol. 100, no. 9, Mar. 2012, Art. ID 113305. 
[21] M. Edalatipour, A. Khavasi, M. Rezaei, and K. Mehrany, "Creation of artificial surface conductivity on metallic metamaterials," J. Lightw. Technol., vol. 30, no. 12, pp. 1789-1794, Jun. 2012.

[22] K. Uchida, T. Noda, and T. Matsunaga, "Spectral domain analysis of electromagnetic-wave scattering by an infinite plane metallic grating," IEEE Trans. Antennas Propag., vol. AP-35, no. 1, pp. 46-52, Jan. 1987.

[23] M. Guglielmi and A. A. Oliner, "Multimode network description of a planar periodic metal-strip grating at a dielectric interface-Part I: Rigorous network formulations," IEEE Trans. Microw. Theory Techn., vol. 37, no. 3, pp. 535-541, Mar. 1989.

[24] M. Guglielmi and A. A. Oliner, "Multimode network description of a planar periodic metal-strip grating at a dielectric interface-Part II: Small-aperture and small-obstacle solutions," IEEE Trans. Microw. Theory Techn., vol. 37, no. 3, pp. 542-552, Mar. 1989.

[25] S. A. Tretyakov and C. R. Simovski, "Dynamic model of artificial reactive impedance surfaces," J. Electromagn. Waves Appl., vol. 17, no. 1, pp. 131-145, Jan. 2003.

[26] R. Rodríguez-Berral, F. Medina, and F. Mesa, "Circuit model for a periodic array of slits sandwiched between two dielectric slabs," Appl. Phys. Lett., vol. 96, Apr. 2010, Art. ID 161104.

[27] R. Rodríguez-Berral, F. Medina, F. Mesa, and M. García-Vigueras, "Quasi-analytical modeling of transmission/reflection in strip/slit gratings loaded with dielectric slabs," IEEE Trans. Microw. Theory Techn., vol. 60, no. 3, pp. 405-418, Mar. 2012.

[28] M. Beruete, M. Navarro-Cía, S. A. Kuznetsov, and M. Sorolla, "Circuit approach to the minimal configuration of terahertz anomalous extraordinary transmission," Appl. Phys. Lett., vol. 98, 2011, Art. ID 014106.

[29] M. Beruete, M. Navarro-Cía, and M. Sorolla, "Understanding anomalous extraordinary transmission from equivalent circuit and grounded slab concepts," IEEE Trans. Microw. Theory Techn., vol. 59, no. 9, pp. 2180-2188, Sep. 2011.

[30] M. Navarro-Cía, P. Rodríguez-Ulibarri, V. Torres, and M. Beruete, "Quarter-wave plate based on dielectric-enabled extraordinary resonant transmission, concepts," IEEE Photon. Technol. Lett., vol. 24, no. 11, pp. 945-949, Jun. 2012.

[31] A. G. Schuchinsky, D. E. Zelenchuk, A. M. Lerer, and R. Dickie, "Full-wave analysis of layered aperture arrays," IEEE Trans. Antennas Propag., vol. 54, no. 2, pp. 1490-502, Feb. 2006.

[32] M. Beruete, I. Campillo, M. Navarro-Cía, F. Falcone, and M. Sorolla, "Molding left- or right-handed metamaterials by stacked cutoff metallic hole arrays," IEEE Trans. Antennas Propag., vol. 55, no. 6, pp. 1514-1521, Jun. 2007.

[33] F. Medina, F. Mesa, and R. Marqués, "Extraordinary transmission through arrays of electrically small holes from a circuit theory perspective," IEEE Trans. Microw. Theory Techn., vol. 56, no. 12, pp. 3108-3120, Dec. 2008.

[34] R. Rodríguez-Berral, F. Mesa, and F. Medina, "Fully analytical circuitlike approach for the TE scattering by narrow-slit printed gratings," in IEEE MTT-S Int. Microw. Symp. Dig., Montreal, QC, Canada, Jun. 2012, pp. 1-3.

[35] R. E. Diaz, J. T. Aberle, and W. E. McKinzie, "TM mode analysis of a sievenpiper high-impedance reactive surface," in Proc. IEEE Antennas Propag. Soc. Int. Symp., Salt Lake City, UT, 2000, pp. 327-330.

[36] J. B. Pendry, L. Martín-Moreno, and F. J. Garcia-Vidal, "Mimicking surface plasmons with structured surfaces," Science, vol. 305, pp. 847-848, Aug. 2004.

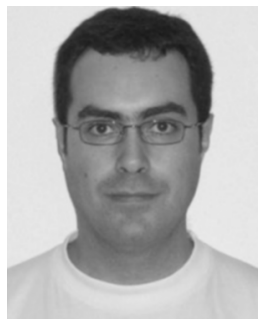

Raúl Rodríguez-Berral was born in Casariche, Seville, Spain, in 1978. He received the Licenciado (M.Sc.) and Ph.D. degrees in physics from the University of Seville, Seville, Spain, in 2001 and 2008 , respectively.

In January 2002, he joined the Department of Applied Physics 1, University of Seville, where he is currently an Assistant Professor. His research interests include the study of the spectrum and the excitation of periodic and nonperiodic planar structures and high-frequency circuit modeling.

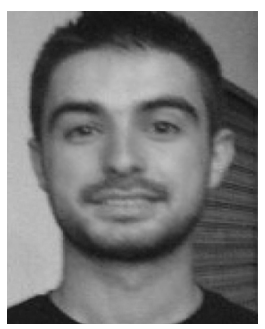

Caros Molero was born in Las Navas, Seville, Spain, in 1987. He received the Licenciado degree in physics from the University of Seville, Seville, Spain, in 2011, and is currently working toward the $\mathrm{Ph} . \mathrm{D}$. degree in applied physics at the University of Seville.

$\mathrm{He}$ is currently with the Department of Applied Physics 1, University of Seville.

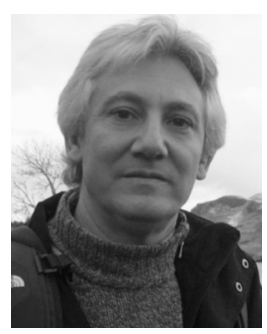

Francisco Medina (M'90-SM'01-F'10) was born in Puerto Real, Cádiz, Spain, in November 1960. He received the Licenciado and Doctor degrees in physics from the University of Seville, Seville, Spain, in 1983 and 1987 respectively.

$\mathrm{He}$ is currently a Professor of electromagnetism with the Department of Electronics and Electromagnetism, University of Seville, and Head of the Microwaves Group. He has coauthored approximately 120 journal papers. His research interest includes analytical and numerical methods for planar structures, anisotropic materials and artificial media modeling.

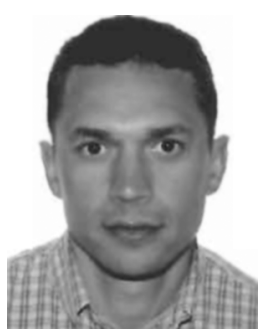

Francisco Mesa (M'93-SM'11) was born in Cádiz, Spain, in April 1965. He received the Licenciado and Doctor degrees in physics from the University of Seville, Seville, Spain, in 1989 and 1991, respectively.

He is currently a Professor with the Department of Applied Physics 1, University of Seville. His research interests focus on electromagnetic propagation/radiation in planar structures. 